\title{
Spontaneous Regression of a Keratoacanthoma Centrifugum Marginatum
}

\author{
Yesul Kim ${ }^{1}$, Klaus F. Helm², Elizabeth M. Billingsley², Charlene Lam²
}

1 Pennsylvania State College of Medicine, Hershey, PA, USA

2 Department of Dermatology, Penn State Hershey Medical Center, Hershey, PA, USA

Key words: keratoacanthoma, squamous cell carcinoma, keratoacanthoma centrifugum marginatum, treatment, management Citation: Kim Y, Helm KF, Billingsley EM, Lam C. Spontaneous regression of a keratoacanthoma centrifugum marginatum. Dermatol Pract Concept. 2019;9(2):157-159. DOI: https://doi.org/10.5826/dpc.0902a16

Accepted: November 26, 2018; Published: April 30, 2019

Copyright: $@ 2019$ Kim et al. This is an open-access article distributed under the terms of the Creative Commons Attribution License, which permits unrestricted use, distribution, and reproduction in any medium, provided the original author and source are credited.

Funding: None.

Competing interests: The authors have no conflicts of interest to disclose.

Authorship: All authors have contributed significantly to this publication.

Corresponding author: Charlene Lam, MD, MPH, 100 Campus Drive, Entrance 3, Suite 100 Hershey, PA 17033 USA. Email: clam@ pennstatehealth.psu.edu

\section{Introduction}

We present an atypical case of keratoacanthoma centrifugum marginatum (KCM) that spontaneously regressed without treatment.

\section{Case Presentation}

A Caucasian man in his 70 s presented with a 3-month history of a large asymptomatic lesion of his left arm. He reported that it initially started as a pinpoint lesion but rapidly grew and stabilized. It was initially biopsied by his primary care physician and read by an outside facility pathologist as a moderately differentiated squamous cell carcinoma (SCC). Physical examination revealed a $6 \times 6 \mathrm{~cm}$, nontender, malodorous plaque with a heaped-up friable tissue rim with clinically normal skin in the center (Figure 1). Lymph node examination was unremarkable. Fungal culture was negative. A punch biopsy for histopathological analysis was performed, showing epithelial fragments and fibrosis, consistent with the diagnosis of a central portion of a KCM (Figure 2). However, the patient subsequently noticed flattening and reduction in size of the lesion. Three months after initial presentation, physical examination showed a $4 \times 5 \mathrm{~cm}$, nontender, nonmalodorous thin plaque with a minor amount of friable tissue and clinically normal skin in the center (Figure 3). It was biopsied in the center and on the periphery to confirm regression, which showed a keratin-filled cystic epithelial invagination and scar, consistent with a regressing KCM (Figure 4). Because of its spontaneous regression, the patient was closely monitored for complete resolution of the lesion. Physical examination 6 months after initial presentation showed a $4 \times 5 \mathrm{~cm}$, nontender, thin pink plaque without friable tissue (Figure 5).

\section{Discussion}

Keratoacanthomas (KAs) are epidermal tumors characterized by rapidly growing, firm, symmetric, dome-shaped nodules with central keratin-filled craters that tend to regress spontaneously [1]. Approximately $98 \%$ of KAs are of the classic, solitary type [2]. Rare variants of solitary KA include subungual KA, giant KA, and KCM. KCM is a very rare variant of KA characterized by progressive peripheral growth with concomitant central healing and atrophy [3]. It was first described by Miedzinski and Kozakiewicz in 1962 [4], and the term keratoacanthoma centrifugum marginatum was coined by Belisario in 1965 [5]. The etiology of KCM is mul- 


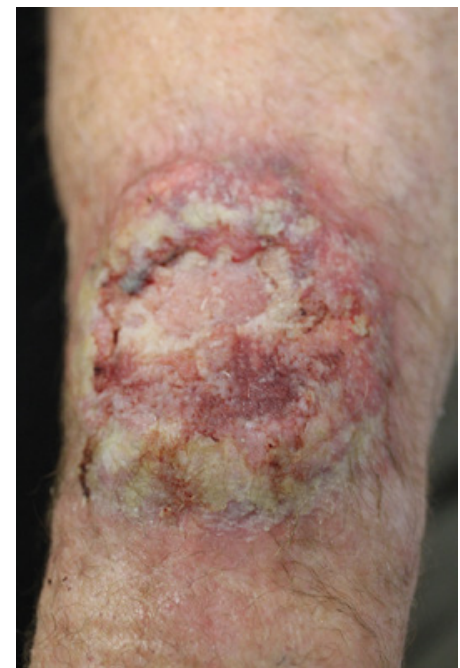

Figure 1. Initial clinical presentation. [Copyright: $\odot 2019$ Kim et al.]

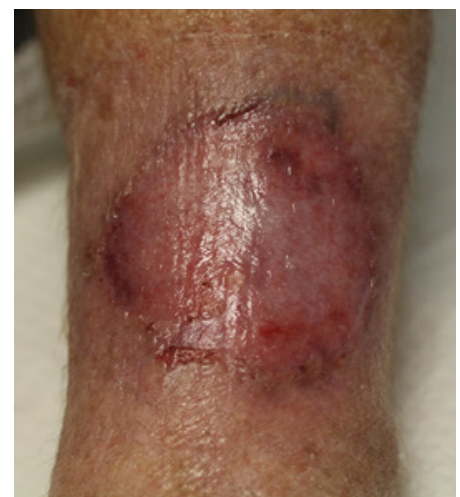

Figure 3. Follow-up clinical presentation 3 months after initial visit. [Copyright: $\odot 2019$ Kim et al.]

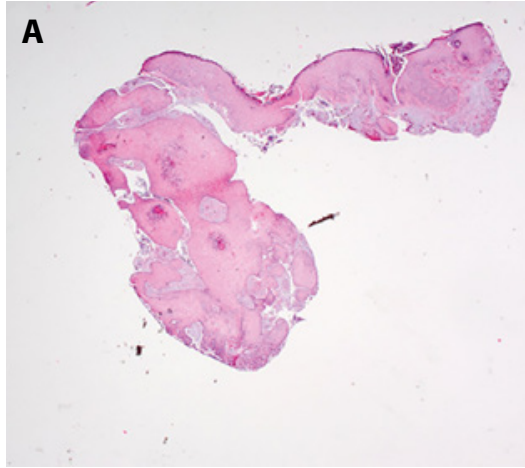

Figure 2. Histopathological examination of the initial biopsy done by the primary care physician and read at an outside facility was interpreted as a moderately differentiated SCC. Hematoxylin and eosin, $\times 2$ (A), $\times 10$ (B), $\times 20$ (C). [Copyright: $\odot 2019$ Kim et al.]
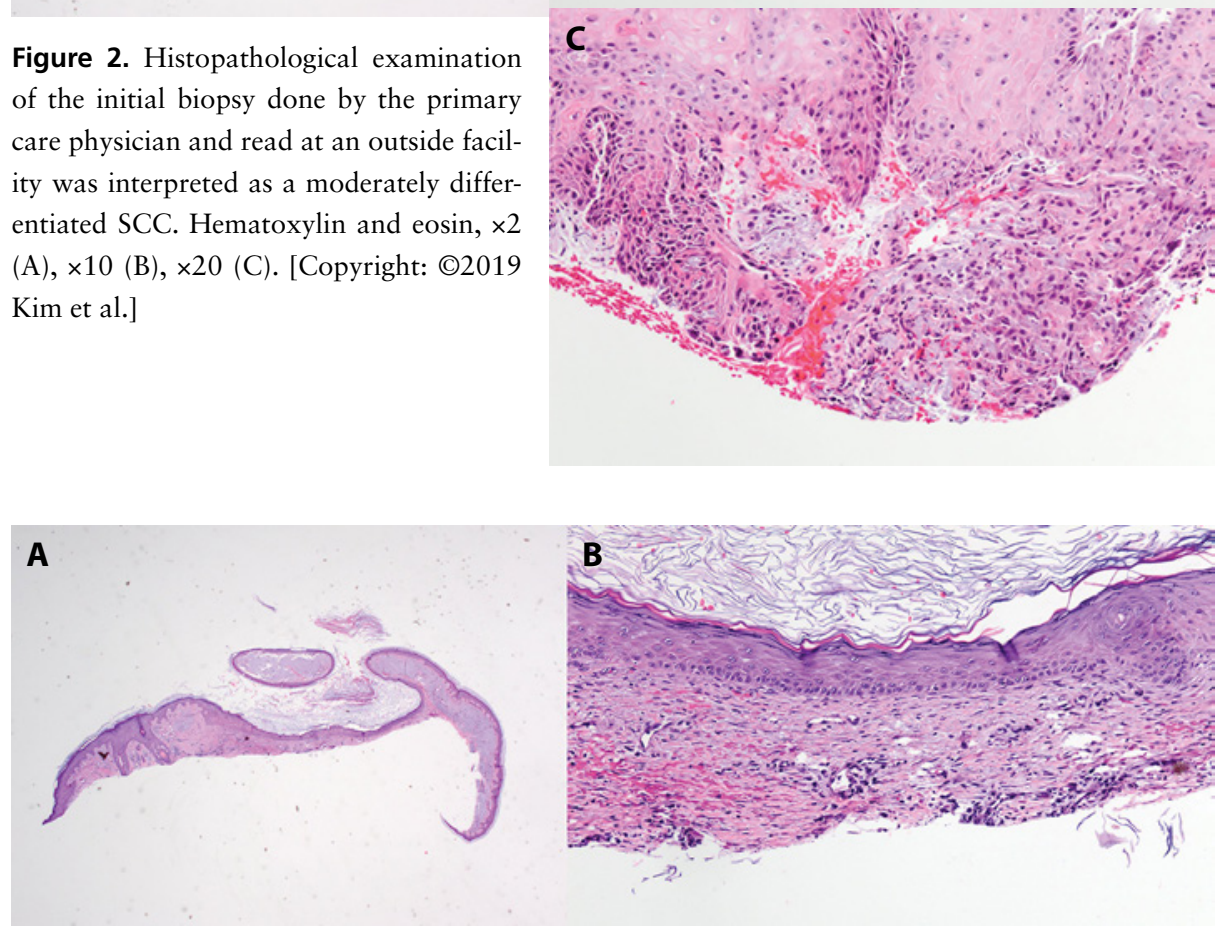

Figure 4. Histopathological examination of the lesion biopsied in the center and on the periphery to confirm regression. It showed keratin-filled cystic epithelial invaginations and scar, consistent with regressing KCM. Hematoxylin and eosin, ×2 (A), ×20 (B). [Copyright: @2019 Kim et al.] tifactorial, including chronic UV light exposure, smoking, and exposure to chemical carcinogens [2]. Histologically, KCM is similar to KA, with a central keratin-filled crater, overhanging lips of epithelium, sharp delineation between tumor nests and stroma, and lack of anaplasia and stromal desmoplasia [6]. KCMs are locally aggressive tumors due to their progressive peripheral expansion. Although they may be locally disfiguring, they are generally benign, with no reported cases of metastasis. KCM is often confused with SCC. Perineural and periadnexal extension may be seen in both entities, but KCM rarely extends beyond the sweat glands [3]. Furthermore, pseudoepitheliomatous hyperplasia and hypergranulosis of the hair follicles occur in the central portion of early KAs but only at the periphery of SCCs [3].

\section{Conclusions}

KCMs, in contrast to the classic solitary KA, do not tend to spontaneously regress [3]. However, we present a case

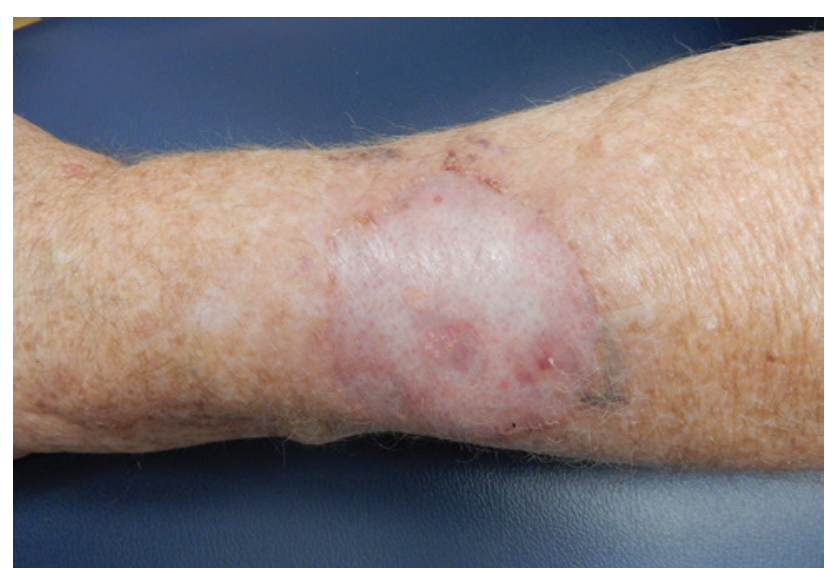

Figure 5. Follow-up clinical presentation 6 months after initial visit. [Copyright: @2019 Kim et al.]

of KCM that spontaneously regressed without intervention and serial biopsies to confirm complete regression. Typically, surgical excision is the preferred therapy for KCM [3]. However, owing to the large size of the lesion in KCM, sur- 
gical excision may result in increased morbidity. Therefore, other modalities of therapies have been used, including oral retinoids, topical 5-fluorouracil, intralesional methotrexate, interferon alfa or bleomycin, cryotherapy, and Mohs micrographic surgery [3].

In conclusion, although KCMs do not typically spontaneously regress, this case of KCM spontaneously regressed without treatment. SCCs can be similar in clinical appearance with dangerous consequences in cases with no treatment; therefore, close monitoring and serial biopsies are paramount to diagnosis and clinical decision-making in KCMs.

\section{References}

1. Cerroni L, Kerl H. Keratoacanthoma. In: Wolff K, Goldsmith LA, Katz SI, et al, eds. Fitzpatrick's Dermatology in General Medicine. 7th ed. New York: McGraw Hill; 2008:1049-1053.
2. Attili S, Attili VR. Keratoacanthoma centrifugum marginatum arising in vitiligo: a case report. Dermatol Online J. 2006;12(2):18.

3. Georgescu T, Oproiu AM, Radasan MG, et al. Keratoacanthoma centrifugum marginatum: an unusual clinical and histopathological diagnostic pitfall. Rom J Morphol Embryol. 2017;58(2):561-555.

4. Miedzinski F, Kozakiewicz J. Das Keratoakanthoma centrifugumeine besondere Varietät des Keratoakanthoms [Keratoacanthoma centrifugum-a special variety of keratoacanthoma]. Hautarzt. 1962;13:348-352.

5. Belisario JC. Brief review of keratoacanthomas and description of keratoacanthoma centrifugum marginatum, another variety of keratoacanthoma. Aust J Dermatol. 1965;8(2):65-72.

6. V'Ickova-Laskoska MT, Laskoski DS. Keratoacanthoma centrifugum marginatum: a rare atypical variant of keratoacanthoma. Clin Exp Dermatol. 2008;33(3):259-261. 